\title{
Signaling Pathways in Osteoclast-driven Bone Metastasis: A mini-review
}

\author{
Manh Tien Tran ${ }^{1, *}$ \\ Department of Dental Pharmacology, Graduate School of Medicine, Dentistry and Pharmaceutical Sciences, Okayama \\ University, Okayama 700-8525, Japan
}

*Corresponding author: Manh Tien Tran, Department of Dental Pharmacology, Graduate School of Medicine, Dentistry and Pharmaceutical Sciences, Okayama University, 2-5-1 Shikata-cho, Kita-ku, Okayama, 700-8525, Japan. Tel: 81-80-8094-6474; E-mail: trantienmanh1508@gmail.com

\section{OSTEOCLASTS}

Osteoclasts (OCs), the multinucleated cells derived from the monocyte/macrophage haematopoietic lineage, are responsible degrading bone during skeletal remodeling. OC differentiation, also called osteoclastogenesis, originates from an interaction between the receptor activator of nuclear factor kappa-B ligand (RANKL) and its RANK receptor in OC precursors [1]. Following this interaction, six core signaling cascades including (1) nuclear factor of activated T cells cytoplasmic-1 (NFATc-1); (2) nuclear factor kappa B (NF-кB); (3) phosphatidylinositol 3-kinase (PI3K/Akt); (4) Jun N-terminal kinase (JNK); (5) extracellular signal-regulated kinase (Erk); and (6) p38 mitogen-activated protein kinase (MAPK) are activated, which is prerequisite for OC differentiation [2]. Once formed, mature OCs secrete lytic enzymes such as tartrateresistant acid phosphatase 5 (ACP5/TRAP) [3], Cathepsin K (CTSK) [4] and Metalloproteinases (MMP9 and MMP14) [5] to resorb bone. Besides, binding of monocyte/macrophage colony stimulating factor (MCS-F) to its c-fms receptor is also required for OC survival and differentiation [6].

\section{$\mathrm{BONE}$}

Bone is one of the most common metastatic sites for metastatic cancer cells, originally derived from breast, lung and prostate cancers [7]. Metastatic cancer cells with bone metastasis is a major source of morbidity and mortality; in contrast, it is not frequently detected in the patients until pain, skeletal-related events (SREs) in spine, ribs, pelvis and proximal long bones, pathological fractures and nerve compression syndromes occur [8].

Bone is a dynamic organ that is consisted of different types of embryo-derived cells such as hematopoietic, stromal, endothelial, adipocytes, OBs, OCs, and osteocytes [9]. Principally, two crucial factors abundantly present in the hematopoietic stem cell (HSC) environment are (1) the chemo-attractant stromal derived factor-1 (SDF-1) or C-X-C motif chemokine ligand $12(\mathrm{CXC12})$ and $(2)$ the cell adhesion factor (Annexin2 or ANXA2) [10]. CXCL12 regulates HSC homing to the bone marrow, while ANXA2 is likely involved in HSC binding to the osteoblastic niche, and may act as anchor of CXCL12 and aid in localization to the niche [10]. The disseminated tumor cells (DTCs) could survive in a quiescent state in bone marrow of cancer patients for years. Accumulating clues have illuminated that DTCs exert homing mechanisms, which are similar to those of HSCs, to themselves support their access to bone marrow. CXCL12 that is released by bone marrow stromal cells (fibroblasts and endothelial cells) interacts with $\mathrm{C}-\mathrm{X}-\mathrm{C}$ motif chemokine receptor 4 (CXCR4) that is aberrantly expressed in cancer cells to promote the migratory capacity of cancer cells in bone [11]. Nevertheless, the detailed mechanisms of how CXCR4/CXCL12 interaction enhance tumor growth and/or metastasis in bone are largely unknown.

\section{OC-MEDIATED REGULATION OF BONE METASTASIS}

In the context of bone diseases, OCs are thought to be involved in several bone disorders, such as osteoporosis, Paget's disease, rheumatoid arthritis, and especially cancer metastasis [12]. Over few decades, there have been lots of considerable efforts on elucidation of the regulatory roles of OCs in metastatic diseases that originate from multiple myeloma, prostate cancer, breast cancer, and/or lung cancer $[13,14]$. In fact, many animal and clinical researches have indicated that OCs play a central role in strengthening the occurrence and progression of bone metastasis through a mechanism underlying the release of factors such as interleukin (IL)-1 and transforming growth factor-beta (TGF- $\beta$ ) that stimulate proliferation, differentiation and growth of metastatic cancer cells. On the other hand, metastatic cells also contribute towards bone resorption by releasing a cocktail of cytokines, such as IL-6, IL-8 and IL- 
11 that activate $\mathrm{OC}$ differentiation and maturation, thereby enhancing bone resorption [15]. As a result, OC has been proposed to be a pivotal regulator for the establishment and maintenance of metastatic foci in skeleton.

The entry of cancer cells into the bone milieu initiates to create a complicated network of cell-cell contact. The minimal essential components are OBs, OCs, metastatic cells and the mineralized bone matrix. Metastatic cells produce a variety of growth factors, most significantly parathyroid hormone-related protein (PTHrP) [16]. The role of PTHrP in bone metastasis with cancer cells is not wholly understood. However, it is best characterized that PTHrP upregulates RANKL and downregulates osteoprotegerin (OPG), the latter is an OBsecreted decoy receptor negatively regulating bone resorption [17]. In overall, growth factors [(TGF- $\beta$, vascular endothelial growth factor (VEGF), insulin-like growth factors (IGFs), and bone morphogenic proteins and fibroblasts-derived factors, $\left.\mathrm{Ca}^{2+}\right]$ that are released into the bone microenvironment enable tumor cells to survive, proliferate and produce more growth factors and more PTHrP, which subsequently perpetuating vicious cycle of bone metastasis.

\section{SUMMARY}

In summary, a thorough understanding of the mechanisms underlying OC-mediated regulation of survival, proliferation and progression of metastatic tumors in bone will profitably contribute to establishment of drug program development as well as discovery of therapeutic treatments for cancer patients with bone metastasis.

\section{FUNDING}

Not applicable.

\section{REFERENCES}

1. Leibbrandt A, Penninger JM (2009) RANKL/RANK as key factors for osteoclast development and bone loss in arthropathies, Adv Exp Med Biol 649 100-13.

2. Park JH, Lee NK, Lee SY (2017) Current Understanding of RANK Signaling in Osteoclast Differentiation and Maturation, Mol Cells 40(10) 706-713.

3. Halleen JM, Tiitinen SL, Ylipahkala H, Fagerlund KM, Väänänen HK (2006) Tartrate-resistant acid phosphatase 5b (TRACP 5b) as a marker of bone resorption, Clin Lab 52(9-10) 499-509.
4. Zaidi M, Troen B, Moonga BS, Abe E (2001) Cathepsin K, Osteoclastic resorption, and osteoporosis therapy, J Bone Miner Res 16(10) 1747-9.

5. Zhu L, Tang Y, Li XY, Keller ET, Yang J, Cho JS, Feinberg TY, Weiss SJ (2020) Osteoclast-mediated bone resorption is controlled by a compensatory network of secreted and membrane-tethered metalloproteinases, Sci Transl Med 12(529).

6. Ross FP (2006) M-CSF, c-Fms, and signaling in osteoclasts and their precursors, Ann N Y Acad Sci 1068 110-6.

7. Suva LJ, Washam C, Nicholas RW, Griffin RJ (2011) Bone metastasis: mechanisms and therapeutic opportunities, Nat Rev Endocrinol 7(4) 208-18.

8. Tsuzuki S, Park SH, Eber MR, Peters CM, Shiozawa Y (2016) Skeletal complications in cancer patients with bone metastases, Int J Urol 23(10) 825-832.

9. Teti A (2011) Bone development: overview of bone cells and signaling, Curr Osteoporos Rep 9(4) 264-73.

10. Jung Y, Shiozawa Y, Wang J, Patel LR, Havens AM, Song J, Krebsbach PH, Roodman GD, Taichman RS (2011) Annexin-2 is a regulator of stromal cell-derived factor-1/CXCL12 function in the hematopoietic stem cell endosteal niche, Exp Hematol 39(2) 151-166.e1.

11. Reid JC, Tanasijevic B, Golubeva D, Boyd AL, Porras DP, Collins TJ, Bhatia M (2018) CXCL12/CXCR4 Signaling Enhances Human PSC-Derived Hematopoietic Progenitor Function and Overcomes Early In Vivo Transplantation Failure, Stem Cell Reports 10(5) 1625-1641.

12. Wang X, Yamauchi K, Mitsunaga T (2020) A review on osteoclast diseases and osteoclastogenesis inhibitors recently developed from natural resources, Fitoterapia 142104482.

13. Liu L,Fan Y, Chen Z, Zhang Y, Yu J (2020) CaSR Induces Osteoclast Differentiation and Promotes Bone Metastasis in Lung Adenocarcinoma, Front Oncol 10305.

14. Tran MT (2021) Overview of $\mathrm{Ca} 2+$ signaling in lung cancer progression and metastatic lung cancer with bone metastasis, Exploration of Targeted Anti-tumor Therapy.

15. Chen YC, Sosnoski DM, Mastro AM (2010) Breast cancer metastasis to the bone: mechanisms of bone loss, Breast Cancer Research 12(6) 215.

16. Bendre M, Gaddy D, Nicholas RW, Suva LJ (2003) Breast cancer metastasis to bone: it is not all about PTHrP, Clin Orthop Relat Res (415 Suppl) S39-45.

17. Ryser MD, Qu Y, Komarova SV (2012) Osteoprotegerin in bone metastases: mathematical solution to the puzzle, PLoS Comput Biol 8(10) e1002703. 